\title{
DETERMINACIÓN Y CUANTIFICACIÓN DE PLUMBAGINA POR HPLC-UV EXTRAIDA DE Dionaea muscipula E. CULTIVADA in vitro
}

\author{
Jorge Chávez Pérez ${ }^{*(a)}$, Ángel Rodríguez Huamán ${ }^{(a)}$, Lillyan Loayza Gutiérrez ${ }^{(a)}$, \\ Piero Huari Soto ${ }^{(b)}$, José Laguna Runser ${ }^{(b)}$
}

\begin{abstract}
RESUMEN
Dionaea muscipula llamada "venus atrapamoscas", es la única especie del género Dionaea perteneciente al grupo de las "plantas carnívoras" que además de su atractivo ornamental, hoy es estudiada para el aislamiento de metabolitos secundarios, habiéndose encontrado diversos compuestos con actividad directa en diferentes enfermedades. La plumbagina (2-metil5-hidroxi-1,4-naftoquinona) ha sido reportada en diferentes especies de géneros como Plumbago (Plumbaginaceae), Drosera y Dionaea (Droseraceae) y Diospyros (Ebenaceae) describiéndose actividades como antiespasmódica, antibacteriana, antifúngica antiparasitaria, antidolor reumático y anticancerígena.

El objetivo del presente trabajo es desarrollar un método fiable por cromatografía HPLC UV para la determinación de plumbagina. El método presentó una la linealidad $\mathrm{r}^{2}=0.997569$ para concentraciones entre 10 y $50 \mu \mathrm{g} / \mathrm{ml}$, con límites de detección (LOD) y de cuantificación (LOQ) de $0.09 \mu \mathrm{g} / \mathrm{ml}$ y de $0,3 \mu \mathrm{g} / \mathrm{ml}$, respectivamente. La precisión de los resultados fue expresada como un \%RSD de 1,34 para el análisis intradiario y de 5,76 para el análisis interdiario. En la exactitud se obtuvo una recuperación máxima de plumbagina de 94,71 \%. Se concluye que el método propuesto es simple, exacto y preciso y puede ser ensayado para la determinación rápida de plumbagina.
\end{abstract}

Palabras clave: Plumbagina, naftoquinona, HPLC. Dionaea muscipula, planta carnívora.

\footnotetext{
(a) Instituto de Investigación de Bioquímica y Biología Molecular. Universidad Nacional Agraria La Molina. Lima Perú. jchavezp@lamolina.edu.pe

(b) Laboratorio de Biotecnología del Programa de Cereales y Granos Nativos de la Facultad de Agronomía. Universidad Nacional Agraria La Molina. Lima - Perú.
} 


\title{
DETERMINATION AND QUANTIFICATION OF PLUMBAGINA BY HPLC-UV EXTRACTED FROM Dionaea muscipula E. CULTIVATED in vitro
}

\begin{abstract}
Background: Dionaea muscipula called "venus flytrap" is the only species of the genus Dionaea belonging to the group of "carnivorous plants" that besides its ornamental appeal nowadays is studied for the isolation of secondary metabolites, having finding various compounds with direct activity in different diseases. Plumbagin (2-methyl-5-hydroxy-1,4-naphthoquinone), has been reported in different genres such as Plumbago species (Plumbaginaceae), Drosera and Dionaea (Droseraceae) and Diospyros (Ebenaceae) describing activities like antispasmodic, antibacterial, antiparasitic antifungal, anti-rheumatic pain and anti-cancer.

The aim of this study is to develop a method by HPLC UV reliable for the determination of plumbagina. The method presented a linearity $r^{2}=0.997569$ for concentrations between 10 and $50 \mathrm{ug} / \mathrm{ml}$, with limits of detection (LOD) and quantitation (LOQ) of $0.09 \mathrm{~g} / \mathrm{ml}$ and $0.3 \mathrm{ug} / \mathrm{ml}$ respectively. The accuracy of the results was expressed as a\% RSD of 1.34 to 5.76 intra diary and inter diary for analysis. The accuracy in maximum recovery of $94.71 \%$ plumbagina was obtained. It is concluded that the proposed method is simple, accurate and precise and can be tested for the rapid determination of plumbagina.
\end{abstract}

Key words: Plumbagin, naphthoquinone, HPLC. Dionaea muscipula, carnivorous plant.

\section{INTRODUCCIÓN}

Dionaea es un género monotípico conformado por la especie Dionaea muscipula o comúnmente llamada "venus atrapamoscas", una especie perteneciente al grupo de las "plantas carnívoras", cuyo nombre científico está integrado por las siguientes partes: Dionaea: proviene del latín "Diana", que es la diosa griega de la caza; muscipula: proviene del latín "musci", significa "mosca", mientras que "pula" significa "atrapa", así que musipula no es más que "atrapamoscas" escrito en latín; y Ellis: En honor al británico John Ellis, que fue el que describió por primera vez a esta planta en 1770 .

D. muscipula Ellis se encuentra en suelos pobres en nitrógeno y fósforo, son plantas de porte bajo, de crecimiento lento, resistentes al fuego y a temperaturas bajas; sobreviven muy bien en suelos arenosos y de turba húmeda. Aunque se ha trasplantado y cultivado en muchos lugares de todo el mundo con éxito, es nativa de los pantanos costeros del norte y de Carolina del Sur, en los Estados Unidos, específicamente, dentro de un radio de 60 millas de Wilmington, Carolina del Sur ${ }^{1,2}$. 
Tradicionalmente, la propagación se lleva a cabo con semillas, esquejes de hojas, porciones basales de la planta y, de vez en cuando, por la apomixis en el tallo de la flor ${ }^{3}$. El cultivo de tejidos de D. muscipula es la técnica actual de multiplicación más usada por los productores ornamentales.

A pesar de su atractivo ornamental, en los últimos años, se ha venido estudiando al grupo de las plantas carnívoras por las enzimas y actividades relacionadas a la digestión de las presas ${ }^{4,5-7}$, lo que conllevó a estudios de aislamiento de metabolitos secundarios, encontrándose diversos compuestos que tienen efecto directo en enfermedades respiratorias, digestivas y a manera de prevención contra el cáncer ${ }^{8-11}$.

La plumbagina (2-metil-5-hidroxi-1,4-naftoquinona) se encuentra en las hojas, corteza y raíces de diferentes especies de géneros como Plumbago (Plumbaginaceae), Drosera (Droseraceae) y Diospyros (Ebenaceae). Se ha descrito el uso de Plumbago zeylanica en el tratamiento del dolor reumático, en donde la plumbagina se muestra como la responsable de tal efecto. Otras actividades descritas para la plumbagina son como antiespasmódica, antibacteriana, antifúngica antiparasitaria y anticancerígena ${ }^{12}$

\section{PARTE EXPERIMENTAL}

\section{Material y métodos}

Material biológico

El material biológico empleado fueron plantas de Dionaea muscipula E. de siete meses de edad, pertenecientes a la empresa SK Vivero. Los ejemplares fueron cultivados bajo sistema in vitro, con $30 \mathrm{ml}$ de medio Murashige Skoog (MS) modificado, con adición de sacarosa (30 g/l), agar SIGMA (5 g/l) y carbón activado (4 g/l) en frascos de $80 \mathrm{ml}$ de volumen. La condiciones ambientales del cultivo fueron programadas para un fotoperiodo de 16-8 horas de luz/sombra, temperatura de $25^{\circ} \mathrm{C}+/-1{ }^{\circ} \mathrm{C}$ y una humedad del cuarto de incubación del $40 \%$.

Reactivos químicos

- Medio de cultivo Murashige Skoog (MS)

- Sacarosa

- Agar (SIGMA)

- Ácido acético glacial grado HPLC

- Agua ultra pura grado HPLC

- Metanol grado HPLC

- Cloroformo grado HPLC

Instrumentación

El equipo para el análisis cromatográfico por HPLC-UV fue un Hitachi Elite LaChromm, con el Software EZChrom Elite v3.3.2, equipado con bomba L-2130, válvula gradiente baja 
presión L-2130ACC, degasificadora L-2000ACC, Interface USB IFU, horno de columnas L-2350, Inyector manual 7725i-006, mezclador cuaternario de solventes. El detector utilizado fue el Hitachi UV Detector L-2400; a una longitud de onda de $260 \mathrm{~nm}$. Todos los ensayos cromatográficos fueron realizados en el laboratorio de análisis instrumental del Instituto de Investigación en Bioquímica y Biología Molecular de la UNALM, 2016.

Obtención del extracto

Las plantas de Dionaea muscipula cultivadas in vitro fueron colocadas en frascos previamente seleccionados, retirando todo el medio de cultivo excedente de las plantas a través de lavados sucesivos con agua destilada, dejándose secar posteriormente durante 72 horas en una cámara con $35 \%$ de humedad ambiental y bajo sombra, la cual fue instalada en el cuarto de incubación de la empresa SK Vivero. Concluido el tiempo de secado, se pesó una muestra de 1 gramo y se pulverizó en un mortero de porcelana. La muestra fue colocada en un cartucho de filtro para el proceso de extracción en un equipo Soxhlet, empleando como solvente cloroformo grado HPLC y realizando 10 ciclos de extracción durante dos horas. Terminado el proceso de extracción se recuperó el solvente por destilación, hasta dejar una muestra de $92 \mathrm{ml}$, la cual fue empleada para el análisis cromatográfico por HPLC. La muestra fue almacenada bajo sombra y a $-20^{\circ} \mathrm{C}$.

\section{Cuantificación de Plumbagina por Cromatografía Líquida de Alta Resolución}

Preparación de los estándares

La solución stock de Plumbagina $(1 \mathrm{mg} / \mathrm{mL})$ fue preparada con metanol grado HPLC y almacenada en oscuridad a $4{ }^{\circ} \mathrm{C}$. A partir de esta solución se prepararon los diferentes puntos de calibración $(10,20,30,40$ y $50 \mu \mathrm{g} / \mathrm{ml}$. Las soluciones fueron filtradas mediante un filtro de membrana de teflón de 0,45 $\mu \mathrm{m}$ antes de ser inyectado en el equipo de HPLC. Cada concentración fue inyectada seis veces en el HPLC-UV, analizándose los coeficientes de correlación $(\mathrm{r})$, coeficiente de determinación $\left(\mathrm{r}^{2}\right)$ y la ecuación de la recta.

Preparación de la muestra

El extracto se concentró en un rotavapor marca Büchi modelo R-3000, durante 1 hora a 40 ${ }^{\circ} \mathrm{C}$. El extracto concentrado se resuspendió en metanol HPLC y se llevó a aforó hasta un volumen de $50 \mathrm{ml}$.

Análisis cualitativo y cuantitativo

El análisis cualitativo del analito se realizó en base al tiempo de retención del pico cromatográfico del estándar puro con una ventana de retención de $5 \%$. Para el análisis cuantitativo se estableció una correlación entre el área del pico cromatográfico y la concentración. Los resultados de concentración fueron expresados como microgramo por 100 gramos de muestra seca $(\mu \mathrm{g} / 100 \mathrm{~g})$.

Linealidad

La linealidad se evaluó mediante el análisis de la relación entre la concentración de plumbagina y la absorbancia reportada por el detector UV. El coeficiente de determinación 
$\left(\mathrm{r}^{2}\right)$ se calculó por ajuste de mínimos cuadrados. Para la realización de la curva de calibración, cada punto se inyectó por quintuplicado.

Límites de detección y cuantificación

Los límites de detección (LODs) y cuantificación (LOQs) fueron calculados usando las expresiones $36 \mathrm{\sigma} / \mathrm{s}$ y $10 \mathrm{\sigma} / \mathrm{s}$, respectivamente; en donde " $\sigma$ " es la desviación estándar interceptada y "s" es la pendiente de la curva de calibración.

\section{Precisión}

La precisión del método indica el grado de dispersión entre los resultados obtenidos de la determinación del analito en una misma muestra. Tres concentraciones de solución estándar de plumbagina $(10,30$ y $50 \mu \mathrm{g} / \mathrm{ml})$ fueron analizados en el mismo día (repetibilidad). Asimismo, una concentración de estándar fue analizada en dos días por diferentes analistas (precisión intermedia) y se determinó la desviación estándar relativa (\% RSD). Las tres muestras de cada nivel fueron preparadas e inyectadas en el HPLC por quintuplicado.

\section{Exactitud}

La exactitud se reportó como el porcentaje de recuperación. Este parámetro muestra la proximidad entre el valor obtenido de forma experimental y el valor real calculado, asegurando que no se produzcan pérdidas durante el análisis. Para ello, se evaluó una muestra diluida 1:2, adicionándole $15 \mu \mathrm{g} / \mathrm{ml}$ de estándar. La muestra se inyectó por quintuplicado en el equipo de HPLC. El Porcentaje de recuperación es igual a la diferencia de los valores mostrados para el estándar con muestra y la muestra por 100 entre el valor añadido de estándar.

\section{RESULTADOS Y DISCUSIÓN}

Condiciones cromatográficas

El análisis cromatográfico del estándar de plumbagina fue realizado con una fase móvil isocrática formada por ácido acético glacial 0.1 M en agua y metanol 33:67 (v/v), la columna empleada fue Waters Bridge Columns ${ }^{\circledR}$ X-Bridge TM C-18 (4.6 x 250 mm, $\left.5 \mu \mathrm{m}\right)$, flujo de $0,75 \mathrm{ml} / \mathrm{min}$. El volumen de inyección de todas las muestras y estándares fue de $20 \mu \mathrm{l}$ con un tiempo de resolución del cromatograma de 10 minutos.

Análisis cualitativo

Se determinó que el estándar de plumbagina se encuentra bien definido con un tiempo de retención de 8,83 minutos, como se observa en el cromatograma de la figura 1 . 


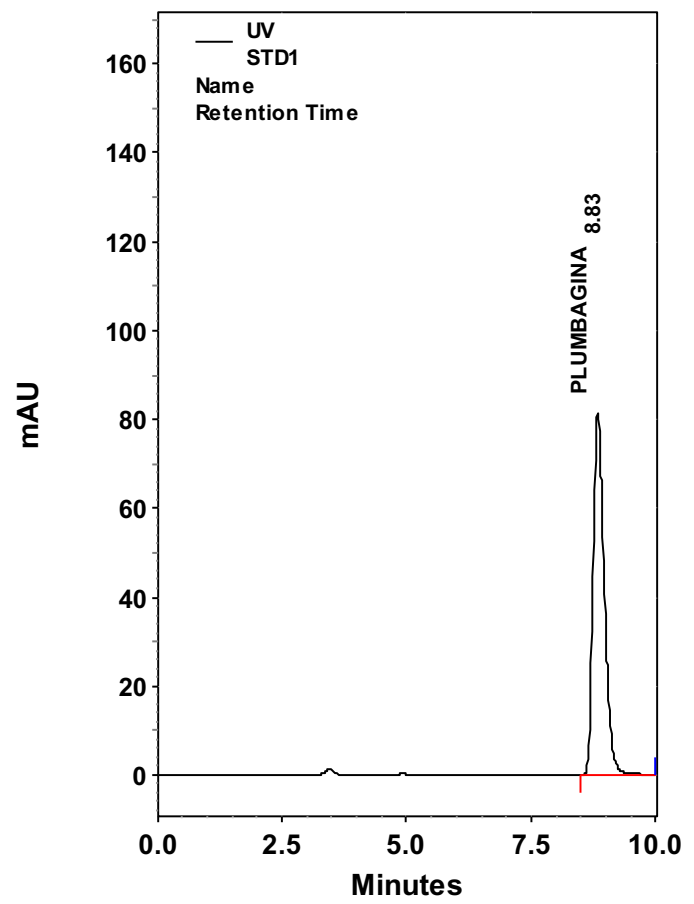

Figura 1. Cromatograma del estándar de plumbagina y determinación del tiempo de retención.

Linealidad: Como se observa en la figura 2, la curva de calibración de plumbagina presenta un valor de coeficiente de determinación $\mathrm{r} 2$ de 0.997569 para concentraciones entre 10 y 50 $\mu \mathrm{g} / \mathrm{ml}$.

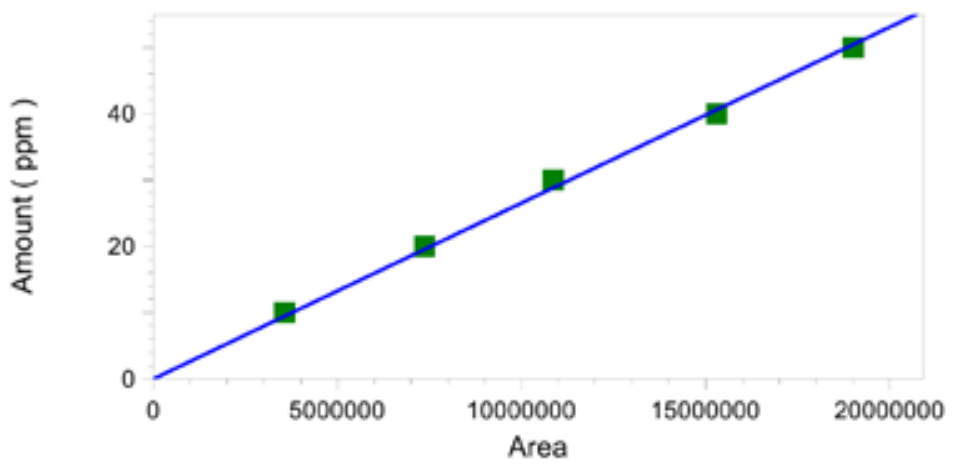

plumbagin (tV)

Average RE: 2.70583e-006 RF StDev: 8.32099e-008 RF 8RSD: 3.07521

Scaling: None LSQ Weighting: None Force Through Zero: On

Replicate Mode: Replace

Eit Type: Linear

$y=2.65446 \mathrm{e}-006 \mathrm{x}+0.000000$

Goodiness of fit $\left(z^{\wedge} 2\right): 0.997569$

Figura 2. Curva de calibración de Plumbagina 


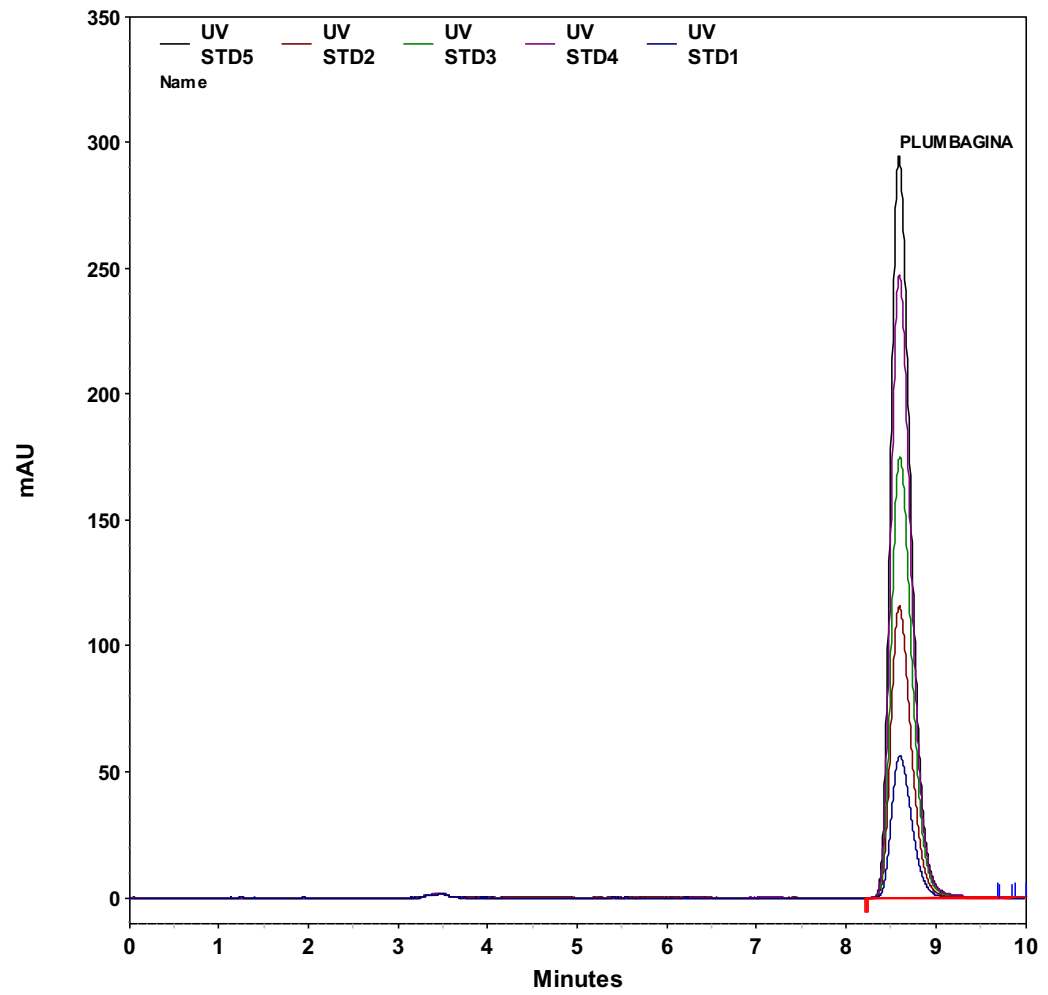

Figura 3. Perfil cromatográfico de plumbagina a diferentes concentraciones. Se observa superposición de los picos de cinco diferentes concentraciones de plumbagina en el mismo tiempo de retención

Límites de detección y cuantificación

Para plumbagina, el límite de detección determinado (LOD) es $0.09 \mu \mathrm{g} / \mathrm{ml}$ y el límite de cuantificación (LOQ) es $0,3 \mu \mathrm{g} / \mathrm{ml}$.

\section{Precisión}

La precisión de los resultados del análisis de plumbagina fue expresada como desviación estándar relativa (\%RSD), obteniéndose valores de 1,34 para el análisis intradiario (repetibilidad) y de 5,76 para el análisis interdiario (precisión intermedia).

\section{Exactitud}

Se obtuvo un porcentaje de recuperación máxima de plumbagina de 94,71\% en la cuantificación del contenido de plumbagina.

Expresión de resultados

La concentración de plumbagina en la muestra se calcula mediante la siguiente expresión: 
$\mu \mathrm{g}$ de plumbagina $/ 100 \mathrm{~g}$ de muestra seca $=\mathrm{LHPLC} * \mathrm{~F} * 100 / \mathrm{Co}$

Donde:

$\mathrm{L}_{\mathrm{HPLC}}: \mu \mathrm{g} / \mathrm{ml}$ plumbagina medidos en el HPLC

F: Factor de dilución ejm: 1 en $10=10$

Co: Concentración del extracto a analizar en $\mathrm{g} / \mathrm{ml}$

\section{CONCLUSIONES}

La necesidad de encontrar nuevas estructuras químicas con actividades biológicas más selectivas y con menores efectos adversos, requiere de ensayos y metodologías validadas en su simpleza, exactitud y precisión y que puedan ser aplicadas para la determinación y cuantificación de estos biocompuestos. Los resultados en el método por HPLC de linealidad, ( $\mathrm{r} 2=0.997569$ para concentraciones entre 10 y $50 \mu \mathrm{g} / \mathrm{ml})$, con límites de detección y límites de cuantificación (con valores de $0,09 \mu \mathrm{g} / \mathrm{ml}$ y $0,3 \mu \mathrm{g} / \mathrm{ml}$ para LOD y LOQ, respectivamente) y la precisión representada en porcentajes de RSD de 1,34 para la repetibilidad (análisis intradiario) y de 5,76 para la precisión intermedia (análisis interdiario), describen un método adecuado para la determinación y cuantificación de plumbagina por cromatografía por HPLC-UV con un porcentaje de recuperación máxima de plumbagina de 94,71\%. Por lo tanto, el método puede ser aplicado para la determinación rápida de plumbagina.

\section{AGRADECIMIENTO}

El presente trabajo fue realizado gracias al apoyo de INNOVATE PERÚ, con el proyecto PIMEN - N573 - 2015.

\section{BIBLIOGRAFÍA}

1. Brittnacher J. Growing Dionaea muscipula. [Internet]. International Carnivorous Plant Society; 2016. [actualizado Jun 2016]. Disponible en: http://www.carnivorousplants. org/howto/GrowingGuides/Dionaea.php

2. Anchor J. Stealing Venus Flytrap plants now a felony [Internet]. Wilmington, North Caroline; 18 set 2014. [actualizado Jun 2016]. Disponible en: http://www.wect.com/ story/26320766/stealing-venus-flytrap-plants-now-a-felony

3. Pietropaolo J, Pietropaolo P. Carnivorous Plants of the World. Portland, Oregon: Timber Press; 1986.

4. Mozingo H, Klein P, Zeevi Y, Lewis E. Venus Flytrap observations by scanning electron microscopy. Am Bot. 1970; 57: 593-598.

5. Robbins R. The nature of the stimuli causing digestive juice secretion in Dionaea muscipula Ellis (Venus's Flytrap). Planta. 1976; 128: 263-265. 
6. Scala J, Iott K, Schwab D, Semersky F. Digestive secretion of Dionaea muscipula (Venus's-Flytrap). Plant Physiol. 1969; 44: 367-371.

7. Slack A. Carnivorous Plants. Sidney: Doubleday Australia; 1981.

8. Banasiuk R, Kawiak A, Krolicka A. In vitro cultures of carnivorous plants from the Drosera and Dionaea genus for the production of biologically active secondary metabolites. BioTechnologia. 2012; 93(2): 87-96.

9. Finnie J, Van Staden J. Drosera spp. (sundew): Micropropagation and in vitro production of plumbagin. Biotechnol Agric For. 1993; 24:164-177.

10. Gaascht F, Dicato M, Diederich M. Venus Flytrap (Dionaea muscipula Solander ex Ellis) Contains Powerful Compounds that Prevent and Cure Cancer. Front Oncol. 2013; 3:202. doi: 10.3389/fonc.2013.00202.

11. Pakulski G, Budzianowski J. Ellagic acid derivatives and naphthoquinones of Dionaea muscipula from in vitro cultures. Phytochemistry. 1996; 4: 775-778.

12. Hsieh YJ, Lin LC, Tsai TH. Determination and identification of plumbagin from the roots of Plumbago zeylanica L. by liquid chromatography with tandem mass spectrometry. J Chromatogr A. 2005; 1083(1-2):141-5. 